\title{
Serosal reaction in chronic gastric ulcers: an immunohistochemical and ultrastructural study
}

\author{
M A Pitt, N Y Haboubi
}

\begin{abstract}
Aim-To study the serosal reaction in gastrectomy specimens with benign chronic peptic ulcers.

Methods-Gastrectomy specimens were fixed in neutral buffered formalin and paraffin wax, and examined using immunohistochemical and ultrastructural techniques.

Results-Nine of the 22 (41\%) cases examined showed reactive hyperplasia of the serosal cells. The cells were predominantly spindle-shaped and were positive on staining with Cam 5.2, AE1/3 and for vimentin. Eight of nine were also positive for smooth muscle actin and five for desmin. Ultrastructural analysis of these cells suggests that they may be myofibroblastic in nature.

Conclusions-These results support the concept of "multipotential" subserosal cells which may differentiate into surface serosa or, in the reactive state, modulate the thin cytoskeletal filaments and become more myofibroblastic. Particular care should be taken not to confuse these reactive serosal cells with carcinoma or smooth muscle tumours in endoscopic biopsy specimens.

(F Clin Pathol 1995;48:226-228)
\end{abstract}

Keywords: Gastric ulcer, serosa, intermediate filaments.

Reactive and neoplastic serosal lesions exhibit diverse growth patterns and express different combinations of cytoskeletal filaments. ${ }^{12}$ This diversity has lead to the hypothesis that surface mesothelial cells, reactive subserosal cells, and both epithelioid and sarcomatoid mesotheliomas originate from multipotential subserosal cells. ${ }^{2}$ Recently, attention has been drawn to the expression of smooth muscle actin and desmin in epitheloid and sarcomatoid mesotheliomas and the reactive serosa in the pleural cavity. ${ }^{34}$

The reactive serosa in the peritoneal cavity was investigated by examining gastrectomy specimens with benign chronic gastric ulcers. Immunohistochemical and ultrastructural techniques were used to assess cytoskeletal expression and to highlight the potential confusion with carcinoma and smooth muscle gastric tumours in biopsy specimens.

\section{Methods}

Twenty two gastrectomy specimens with chronic peptic ulcers were retrieved from the files of Withington Hospital. Specimens were fixed in neutral buffered formalin and a minimum of four blocks processed for embedding in paraffin wax. Sections were stained with haematoxylin and eosin, and reviewed to exclude neoplasia. Those with a complete gastric wall were then selected and stained immunohistochemically using the avidinbiotin peroxidase complex.

Initially, all blocks were examined using Cam 5.2. This revealed 13 cases with absent serosa or a normal serosal monolayer. Lack of serosa in some cases was possibly because of damage sustained during surgery. The remaining nine cases had multilayered Cam positive cells along the gastric serosa, indicative of serosal hyperplasia. These cases were then examined further using serial sections and were stained with periodic acid Schiff (PAS)/diastase, AE1/3, and for smooth muscle actin, carcinoembryonic antigen, and vimentin. Appropriate positive and negative controls were included in each run and the internal control checked.

In one case selected fragments from the paraffin wax block were retrieved from an area of serosal hyperplasia, confirmed by toluidine blue staining and light microscopy. These fragments were deparaffinised in xylene and rehydrated in cacodylate buffer. They were then treated with osmium tetroxide, dehydrated in ethanol and embedded in epoxy resin. Ultrathin sections were stained with uranyl acetate and Reynolds lead citrate.

\section{Results}

On light microscopy, the reactive serosal cells were predominantly spindle-shaped and arranged in sheets and columns. The subserosal sheets varied between three and 10 cells thick and ran parallel to the serosal surface. In two of the nine cases columns of the same spindle cell type were arranged perpendicularly and ran through the muscularis propria and fibrous tissue of the ulcer base towards the mucosal surface reaching the ulcer base, which was characterised by granulation tissue and inflammatory debris with which the spindle cells merged. Areas of serosal hyperplasia were distinguishable from

\author{
General Hology, Bolton \\ Road, Farnworth, Bolton \\ BL4 0JR. \\ Accepted for publication \\ 7 June 1994 \\ Department of \\ South Manchester, \\ M A Pitt
}



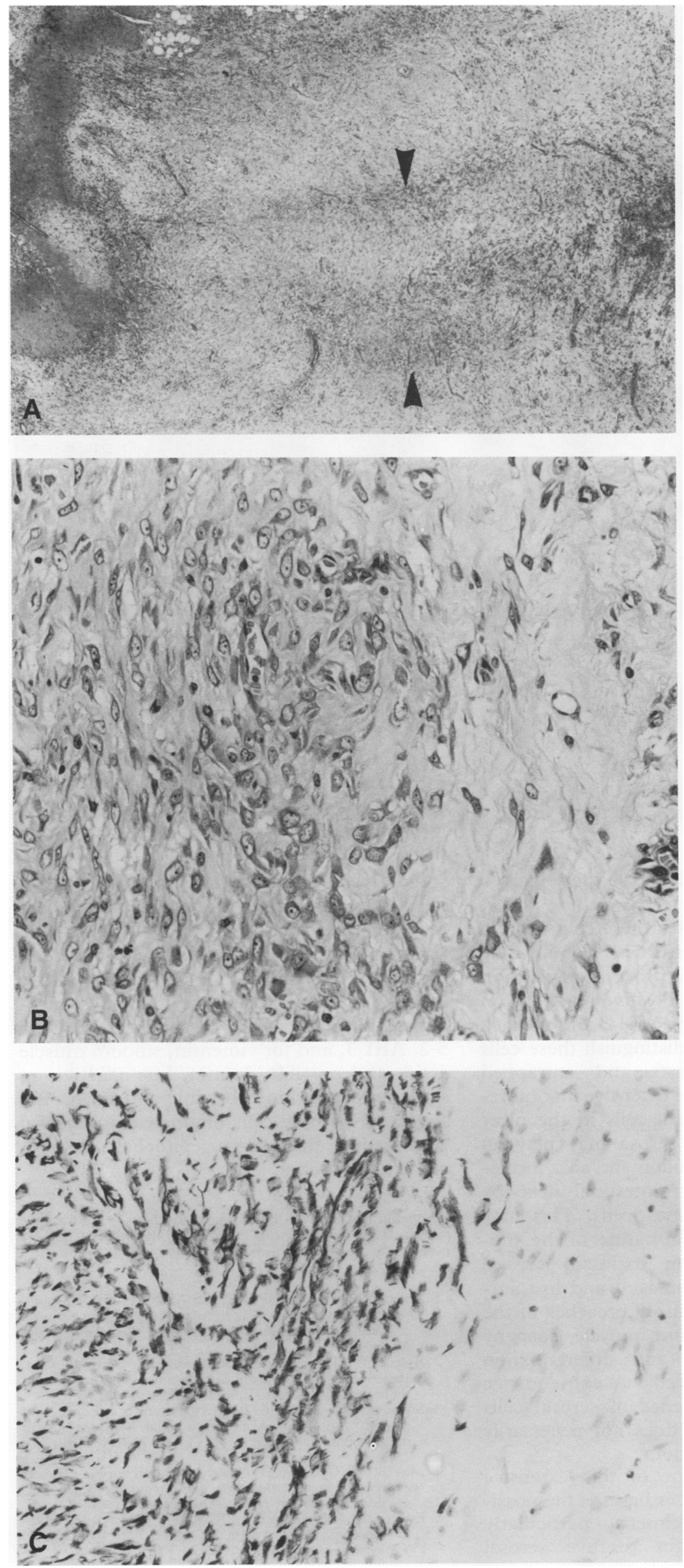

Figure 1 A: Florid reactive serosal hyperplasia in the base of an ulcer with perpendicular extension (arrowheads). B: An area of serosal hyperplasia (left) with adjacent stroma. Note the differing cellularity and some polygonal serosal cells.

C: Cam 5.2 immunostaining of serosal cells. Note the absence of staining in adjacent stroma. adjacent fibrous scar tissue and the smooth muscle coat by their greater cellularity and continuity with the underlying serosa (figs $1 \mathrm{~A}$ and 1B). Serosal cells had larger, paler nuclei than adjacent stromal cells and some were more polygonal with an epithelioid appearance (fig 1B). Serosal cells were negative on staining with PAS/diastase.

In all nine cases the serosal spindle cells were Cam 5·2, $\mathrm{AE} 1 / 3$ and vimentin positive (fig 1C). Eight of the nine cases were smooth muscle actin positive, while five of the nine were desmin positive. The one smooth muscle actin negative case was also desmin negative. Generally, staining with Cam $5 \cdot 2, \mathrm{AE} 1 / 3$, and for vimentin and smooth muscle actin was strongly positive, whereas desmin was only weakly or focally positive. All cases were negative on staining for carcinoembryonic antigen. The adjacent fibrous scar tissue and smooth muscle coat was Cam 5.2 and AE1/3 negative.

Electron microscopy of the reactive serosal cells from one case revealed spindle cells scattered within a collagenous matrix with numerous thin-walled vessels (fig $2 \mathrm{~A}$ ). Spindle cells were fibroblastic in appearance with abundant rough endoplasmic reticulum, collagen secreting granules, and adjacent extracellular collagenous matrix. Longitudinal aggregations of cytoplasmic filaments were also seen, some of which contained focal densities (fig 2B). No external lamina or fibronexus junctions could be identified. Occasional centrioles and junctional complexes were also seen.

\section{Discussion}

Reactive serosa exhibits both cytokeratins and vimentin, while normal surface mesothelial cells are cytokeratin positive but vimentin negative, and normal subserosal cells are vimentin positive only. Subserosal cells have ultrastructural features similar to those of myofibroblasts. ${ }^{12}$ Recently, mesotheliomas and the reactive pleura have been found to express desmin and smooth muscle actin. ${ }^{34}$ In this study, the reactive peritoneal serosa also expressed cytokeratin and vimentin and, like the pleura, often expressed the intermediate filament desmin and smooth muscle actin. Electron microscopy confirmed that the spindle cells had some myofibroblastic features with abundant rough endoplasmic reticulum, collagen production and focal densities, although fibronexus junctions were absent. ${ }^{5}$ These observations support the concept of "multipotential" subserosal cells, which are capable of forming surface mesothelial or spindle cells, showing "myofibroblastic" immunohistochemical and electron microscopic features when suitably stimulated.

The extent of serosal hyperplasia was variable, as shown by proliferation of spindle cells from the serosa with the characteristic immunophenotype. This variability, and the absence of hyperplasia in some cases, may be related to factors such as ulcer chronicity and depth. It might be expected that the deeper 
Figure 2 A: Spindle cell is present in a collagenous matrix with fibroblastic features and focal densities (arrowheads) ( $\times 8000)$ and $B$ : at higher magnification ( $\times 50$ 000) showing focal density and cytoplasmic filaments.
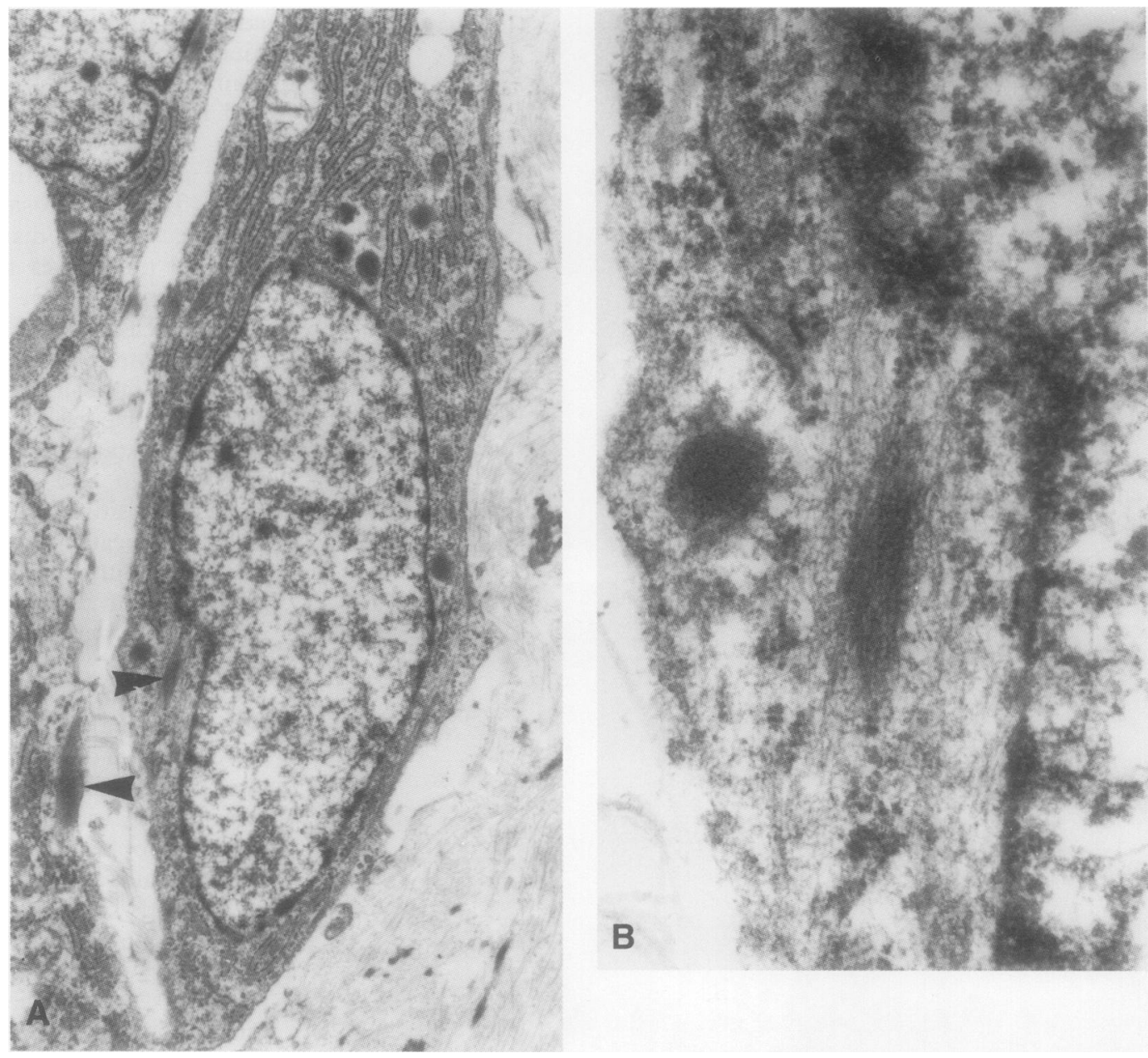

and more chronic the ulcer, the more likely a serosal response.

In two of the nine cases these cells formed continuous columns which extended into the fibrous ulcer base and up to the granulation tissue in the ulcer floor. Positive reactions on staining with Cam 5.2 and AE1/3 and the lack of fibronexus junctions distinguish these cells from scar myofibroblasts and entrapped smooth muscle cells. The extensive incorporation of reactive subserosal cells in the ulcer base suggests that the serosa may play an important role in the healing and scar formation associated with gastrointestinal ulcers by providing "myofibroblastic" cells. This may explain the multipotential nature of the subserosal cells which can maintain serosal integrity by replacing damaged and lost surface cells as well as repairing breaches of the underlying adventitia and muscle coat by migration, proliferation and differentiation into myofibroblast-like cells. A consequence of this is that the presence of serosal cells within the deep tissues does not necessarily indicate malignant behaviour.

A further consequence of this extensive serosal migration into ulcer bases is the possibility that biopsy specimens, particularly endoscopic, may contain reactive serosal spindle cells. In this situation immunohistochemistry performed to detect cancer cells (cytokeratin positive) or smooth muscle tumour cells (desmin/smooth muscle actin positive), may give rise to false positive results. The use of a panel of antibodies showing spindle cells positive on staining with Cam $5 \cdot 2, \mathrm{AE} 1 / 3$, and for vimentin, smooth muscle actin, and possibly desmin, but negative on staining for carcinoembryonic antigen, would suggest that these cells originate from serosal cells rather than from epithelial or nonepithelial tumours.

We would like to thank Dr A Curry and Miss P L Rowlands for assistance with electron microscopy and Mr P Clark for performing the immunohistochemistry.

1 Bolen JW, Hammar SP, McNutt MA. Reactive and neoand immunocytochemical study. Am $\mathcal{F}$ Surg Pathol 1986; 10:34-47.

2 Bolen JW, Hammar SP, McNutt MA. Serosal tissue: Reactive tissue as a model for understanding mesotheliomas. Ultrastruct Pathol 1987;11:251-62.

3 Scoones DJ, Richman PI. Expression of desmin and smooth muscle actin in mesothelial hyperplasia and mesothelioma [abstract]. I Pathol 1993;169(Suppl): 188 .

4 Mayall FG, Goddard H, Gibbs AR. Intermediate filament expression in mesotheliomas: leiomyoid mesotheliomas are not uncommon. Histopathology 1992;21:453-7.

5 Eyden BP, Christensen L, Tagore V, Harris M. Myofibrosarcoma of subcutaneous soft tissue of the cheek f Submicrosc Cytol Pathol 1992;24:307-13. 